\title{
The Real-World Practice of Fruquintinib for Chinese Patients with Metastatic Colorectal Cancer
}

\author{
Yan Song \\ Tao Qu \\ Honggang Zhang \\ Yongkun Sun (D) \\ Chengxu Cui \\ Yihebali Chi \\ Wen Zhang \\ Xingyuan Wang \\ Lin Yang
}

Department of Medical Oncology, National Cancer Center/National Clinical Research Center for Cancer/Cancer Hospital, Chinese Academy of Medical Sciences and Peking Union Medical College, Beijing, I0002I, People's Republic of China
Background: In FRESCO trial, a phase III study of fruquintinib demonstrated a significant improvement on the overall survival (OS) of patients with metastatic colorectal cancer (mCRC) who failed to response to available standard treatments. The aim of the current study was to evaluate the safety and effectiveness of fruquintinib in Chinese mCRC patients in the real-world setting.

Methods: Patients with mCRC treated with fruquintinib at our hospital were retrospectively reviewed. Patient demographics, treatment, adverse events and survival data were collected. OS and progression-free survival (PFS) were estimated using the Kaplan-Meier method.

Results: In total, 76 patients were evaluated from December 2018 to January 2020. The median (range) age was 59.5 (34-86) years, ECOG PS 0-1/2 was 86.8\%/13.2\%, and 38 $(50 \%) / 30(39.5 \%)$ patients had experienced more than two prior therapies for mCRC. The median treatment duration was 3.6 cycles. Treatment-related adverse events (TRAEs) resulted in dose reduction were $17.1 \%$ of the patients without any treatment discontinuation. The most common grade 3 or 4 TRAEs were hypertension $(9.2 \%)$, hand-foot skin reaction $(7.9 \%)$, thrombocytopenia $(3.9 \%)$, anaemia $(2.6 \%)$, increased ALT (1.3\%), oral mucositis $(1.3 \%)$, proteinuria $(1.3 \%)$ and neutropenia $(1.3 \%)$. The median PFS was 5.1 months $(95 \%$ CI 3.8-6.4 months), and the median OS was 12.0 months (95\% CI 8.0-16.1 months). In patients with hypertension or hypothyroidism, a survival extension approximate to 6 months was observed, but the difference is not yet statistically significant. CEA decreased after fruquintinib treatment could be considered as a potential predictor for better OS.

Conclusion: The outcome of this real-life study was consistent with that of the randomised controlled trial. There were no new safety concerns. Future studies of fruquintinib should be conducted to identify patients who tend to obtain more benefits from fruquintinib alone or in combination with other agents.

Keywords: fruquintinib, metastatic colorectal cancer, real-world settings, toxicities

\section{Introduction}

Colorectal cancer is the third most common cancer worldwide and the second leading cause of death from cancer. It is estimated that there are 1.8 million new cases and around 900,000 deaths in the world in 2018. ${ }^{1}$ Colorectal cancer ranks third in terms of incidence among Chinese, with 28.2 per 100,000 persons, and fifth in mortality, with 13.6 per 100,000 persons. $^{2}$

Owing to novel treatment options, the overall survival (OS) of $\mathrm{mCRC}$ patients is reported to be nearly 32 months. Currently, the standard of care for systemic treatment
Correspondence: Lin Yang; Tao Qu Department of Medical Oncology, National Cancer Center/National Clinica Research Center for Cancer/Cancer Hospital, Chinese Academy of Medical Sciences and Peking Union Medical College, Beijing, I0002I, People's Republic of China

Email linyang@cicams.ac.cn; thomasqu1972@hotmail.com 
for mCRC consists of fluoropyrimidine, irinotecan and oxaliplatin in combination or in sequence with biological agents such as bevacizumab and ziv-aflibercept. For mCRC patients with wild-type RAS and wild-type BRAF, treatment with Cetuximab and panitumumab (monoclonal antibodies against epidermal growth factor receptor) are usually applied. $^{3}$

Advances in prognosis and treatments have provided significant improvement in Overall Survival (OS) for mCRC patients after the failure of second-line therapy. As an oral multikinase inhibitor, regorafenib received FDA approval in 2012 as indicated for heavily pretreated mCRC patients. It was also approved for same indication in China on March 5, 2017. Compared to placebo, treatment with regorafenib significantly increased the median OS (6.4 months vs 5.0 months, hazard ratio [HR] $0.77, p=0.005)$ and the median progression-free survival (PFS) (1.9 vs 1.7 months, HR $0.49, p<0.001)$ in the CORRECT trial. Various AE management strategies for regorafenib, including reducing the dose and altering the dosage schedule, have been evaluated and shown to be effective in different subgroups of patients with mCRC. Trifluridine/tipiracil (TAS-102), an oral nucleoside compound, demonstrated a critical improvement in both the median OS (7.1 vs 5.3 months, HR $0.58, p<$ $0.001)$ and median PFS (2.0 vs 1.7 months, HR $0.48, p<$ $0.001)$ compared to best support care in the RECOURSE trial; it is another drug that was approved for definitive treatment in the third-line setting for $\mathrm{mCRC}$ by the FDA in 2015 and was recently approved in China in July 2020.

Fruquintinib is a potent, highly selective and orally active inhibitor of VEGFR1, 2, and 3 tyrosine kinases. ${ }^{4}$ In the randomised phase III clinical trial FRESCO, among 416 Chinese mCRC patients who progressed after at least 2 prior chemotherapy regimens, oral fruquintinib compared with BSC has demonstrated a statistically significant increase in OS (9.3 vs 6.6 months; HR 0.65; 95\% CI, $0.51-0.83 ; P<0.001)$. The median PFS was also significantly increased by fruquintinib treatment compared with placebo (3.7 vs 1.8 months; HR 0.26 ; 95\% CI, 0.21 to 0.34; $P<0.001$ ). Most frequent grade 3 to 4 adverse events were hypertension $(21.2 \%)$, hand-foot skin reaction $(10.8 \%)$, and proteinuria $(3.2 \%) .^{5}$

Fruquintinib was approved by the CFDA in September 2018 for patients with $\mathrm{mCRC}$ who progressed after two or more lines of standard systemic therapy, containing fluoropyrimidine, oxaliplatin and irinotecan, with or without prior treatment with anti-EGFR or anti-VEGF antibody. However, the criteria for enrolment in clinical trials were stringent, such as age, weight, starting dose, and prior antitumour treatment, and might not represent real-life patients and outcomes. Our study aimed to determine the efficacy and safety profile of fruquintinib in clinical practice and to determine the factors that influence the efficacy of fruquintinib in this group of patients.

\section{Methods}

Under approval from local research ethics board, we conducted a retrospective single-centre study at our institution on mCRC patients with fruquintinib treatment started from December 2018 to January 2020.

Eligible patients for this analysis were those who had histologically confirmed mCRC; who were not suitable for chemotherapy or refused chemotherapy; progressed on all the feasible systemic treatments, including oxaliplatin, irinotecan, fluoropyrimidine, bevacizumab and cetuximab when applicable; and received at least one cycle of fruquintinib. The recorded data included demographics, disease characteristics, prior systemic therapy, laboratory values, efficacy and adverse events. Informed consent was obtained in this trial and all patient data was confidential. This study was performed in accordance with the Declaration of Helsinki and the international standards of good clinical practice. This study has been approved by ethics committee of National Cancer Center/Cancer Hospital, Chinese Academy of Medical Sciences and Peking Union Medical College (Approval number: 20/ 411-2607).

We aimed to represent the clinical profiles of the mCRC patients treated with fruquintinib and to demonstrate the efficacy and toxicity of fruquintinib in a realworld practice. All statistical analyses were performed using SPSS 20.0 (IBM Corp., USA). Survival was estimated using the Kaplan-Meier method. A Cox proportional hazards model was adopted to evaluate the association between the baseline characteristics and OS. Tumour response was evaluated every two cycles according to the Response Evaluation Criteria in Solid Tumours (RECIST) V1.1. OS and PFS were estimated using the Kaplan-Meier method and compared by the Log rank test. Adverse Events were graded according to National Cancer Institute Common Terminology Criteria for Adverse Events (NCI CTCAE) V.5.0. The safety profile, including adverse events of special interest (AESIs) and treatment-emergent adverse events (TEAEs), was analysed. 


\section{Results}

\section{Patient Characteristics}

Seventy-six patients were reviewed in this study. By the data cut-off date of July 15, 2020, the median follow-up was 13.6 months (range 12.0-15.2 months). The median age was 59.5 years (range 34-86 years). The majority of patients were in good conditions (ECOG PS 0-1: 86.8\%). The most common primary site of disease was the rectum $(52.6 \%)$, while the main metastatic sites were the liver (67.1\%), lung (60.5\%), and lymph nodes (40.8\%). Patients with RAS wild-type tumours accounted for approximately $33 \%$, and $74 \%$ of patients had BRAF wild-type tumours. About $39.5 \%$ of patients were treated with fruquintinib as the fourth-line and beyond. The percentages of the patients treated with bevacizumab, cetuximab and regorafenib were $84.2 \%$, $22.4 \%$ and $19.7 \%$, respectively (Table 1 ).

\section{Fruquintinib Treatment}

Sixty-six patients $(86.8 \%)$ initiated fruquintinib treatment at a dose of $5 \mathrm{mg}$ which was the standard daily dose, while the others reduced the daily dose to $4 \mathrm{mg}$ ( 2 patients) and $3 \mathrm{mg}$ (8 patients) when took fruquintinib because they had an ECOG PS of 2. Thirteen patients (17.1\%) reduced the dose at the first or second cycle, and none of the patients required treatment suspension. The doses of seven patients were reduced from $5 \mathrm{mg}$ to $4 \mathrm{mg}$, while those of six patients were reduced to $3 \mathrm{mg}$. The median treatment duration was 3.6 cycles (range 1.0-16.8 cycles). Handfoot skin reaction (HFSR) (seven patients, 53.8\%), hypertension (five patients, 38.5\%) and oral mucositis (one patient, $7.7 \%$ ) were the main reasons for dose reduction.

\section{Safety}

Overall, 70 patients $(92.1 \%)$ had at least one fruquintinibrelated TEAE (Table 2). The most frequent fruquintinibinduced TEAEs were hypertension (48.7\%), HFSR (43.4\%), proteinuria (27.6\%) and hypothyroidism (22.4\%). Grade 3 or 4 fruquintinib-induced TEAEs were observed in $27.6 \%$ of patients, such as HFSR (9.2\%), hypertension (9.2\%), thrombocytopenia (3.9\%) and anaemia (2.6\%). Overall, no treatment-induced deaths were reported.

\section{Effectiveness}

The median OS was 12.0 months (95\% CI $8.0-16.1$ months) (Figure 1). The 6-month and 1-year OS rates
Table I Baseline Characteristics

\begin{tabular}{|c|c|}
\hline Characteristics & $\mathbf{N}=76, \mathbf{N}(\%)$ \\
\hline \multicolumn{2}{|l|}{ Age (years) } \\
\hline Median (range) & $59.5(34-86)$ \\
\hline$<60$ & $38(50.0)$ \\
\hline$\geq 60$ & $38(50.0)$ \\
\hline \multicolumn{2}{|l|}{ Sex } \\
\hline Male & $40(52.6)$ \\
\hline Female & $36(47.4)$ \\
\hline \multicolumn{2}{|l|}{ ECOG } \\
\hline $0-1$ & $66(86.8)$ \\
\hline 2 & $10(13.1)$ \\
\hline \multicolumn{2}{|l|}{ Primary site of disease } \\
\hline Rectum & $40(52.6)$ \\
\hline Sigmoid colon & $16(21.1)$ \\
\hline Descending colon & $5(6.6)$ \\
\hline Transverse colon & $\mathrm{I}(\mathrm{I} .3)$ \\
\hline Ascending colon & $14(18.4)$ \\
\hline \multicolumn{2}{|l|}{ Metastatic sites } \\
\hline Liver & $51(67.1)$ \\
\hline Lung & $46(60.5)$ \\
\hline Lymph node & $31(40.8)$ \\
\hline Peritoneum & $17(22.4)$ \\
\hline Bone & $4(5.3)$ \\
\hline Ovary & $2(2.6)$ \\
\hline \multicolumn{2}{|l|}{ Primary tumor surgery } \\
\hline Yes & $53(69.7)$ \\
\hline No & $23(30.3)$ \\
\hline \multicolumn{2}{|l|}{ RAS status } \\
\hline Wild-type & $25(32.9)$ \\
\hline Mutant & $34(44.7)$ \\
\hline Unknown & $17(22.4)$ \\
\hline \multicolumn{2}{|l|}{ BRAFV600E status } \\
\hline Wild-type & $56((73.7)$ \\
\hline Mutant & $\mathrm{I}(\mathrm{I} .3)$ \\
\hline Unknown & $19(25.0)$ \\
\hline \multicolumn{2}{|l|}{ MMR status } \\
\hline PMMR & $51(67.1)$ \\
\hline dMMR & $2(2.6)$ \\
\hline Unknown & $23(30.3)$ \\
\hline \multicolumn{2}{|l|}{ Number of prior regimens for metastatic disease } \\
\hline 0 & $2(2.6)$ \\
\hline I & $6(7.9)$ \\
\hline 2 & $38(50.0)$ \\
\hline 3 & $20(26.3)$ \\
\hline$\geq 4$ & $10(13.2)$ \\
\hline
\end{tabular}

(Continued) 
Table I (Continued).

\begin{tabular}{|l|c|}
\hline Characteristics & N=76, N (\%) \\
\hline Prior chemotherapy & \\
Oxaliplatin & $65(85.5)$ \\
Irinotecan & $70(92.1)$ \\
Capecitabine & $70(92.1)$ \\
5-Fu & $27(35.5)$ \\
S-I & $24(31.6)$ \\
Raltitrexed & $36(47.4)$ \\
\hline Prior targeted therapy & \\
Bevacizumab & $64(84.2)$ \\
Cetuximab & $17(22.4)$ \\
Regorafenib & $15(19.7)$ \\
\hline
\end{tabular}

Table 2 Fruquintinib-Induced Adverse Events

\begin{tabular}{|l|c|c|}
\hline Adverse Events & Any grade N (\%) & Grade $3 / 4$ N (\%) \\
\hline Any event & $70(92.1)$ & $2 I(27.6)$ \\
Hypertension & $37(48.7)$ & $7(9.2)$ \\
Hand-foot skin reaction & $33(43.4)$ & $7(9.2)$ \\
Proteinuria & $21(27.6)$ & $1(1.3)$ \\
Hypothyroidism & $17(22.4)$ & $0(0)$ \\
Diarrhea & $14(18.4)$ & $0(0)$ \\
Oral mucositis & $13(17.1)$ & $1(1.3)$ \\
Fatigue & $13(17.1)$ & $0(0)$ \\
Hoarseness & $12(15.8)$ & $0(0)$ \\
Decreased appetite & $9(11.8)$ & $0(0)$ \\
Thrombocytopenia & $8(10.5)$ & $3(3.9)$ \\
AST increase & $6(7.9)$ & $0(0)$ \\
Gastritis & $6(7.9)$ & $0(0)$ \\
Leukopenia & $3(3.9)$ & $0(0)$ \\
Neutropenia & $3(3.9)$ & $I(1.3)$ \\
ALT increase & $3(3.9)$ & $I(1.3)$ \\
Bilirubin increase & $3(3.9)$ & $0(0)$ \\
Bleeding & $3(3.9)$ & $0(0)$ \\
Anemia & $2(2.6)$ & $2(2.6)$ \\
\hline
\end{tabular}

were $73.7 \%$ and $51.4 \%$, respectively. The median PFS was 5.1 months (95\% CI 3.8-6.4 months) (Figure 2).

The median overall survival was 11.3 months (95\% CI 5.9-16.8 months) for the standard-dose group (53 patients, dose starting from $5 \mathrm{mg} /$ day without reductions during treatment) and 13.7 months (95\% CI 8.0-16.1 months) for the low-dose group (23 patients, dose starting from 3 to $4 \mathrm{mg}$ /day or reduced from $5 \mathrm{mg}$ /day for any reason during treatment $)(\mathrm{HR}=0.77,95 \%$ CI $0.38-1.59, \mathrm{P}=0.48)$. For patients who were treated with fewer prior regimens showed a trend towards longer survival. The median OS of patients who received fruquintinib as first/second-line,

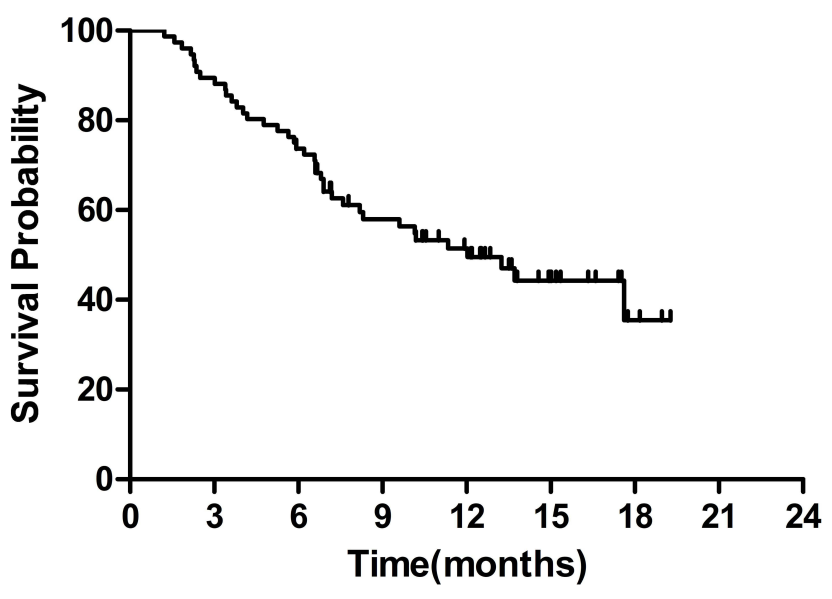

Figure I Kaplan-Meier survival curves of overall survival.

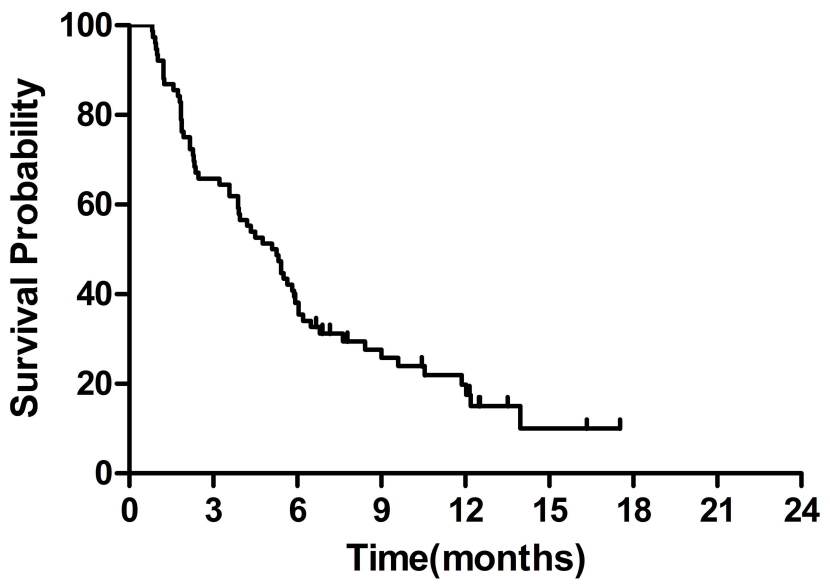

Figure 2 Kaplan-Meier survival curves of progression-free survival.

third-line or fourth-line treatment and beyond was NR (not reached), 13.2 months or 6.9 months, respectively ( $\mathrm{HR}=1.30,95 \% \mathrm{CI} 0.78-2.15, \mathrm{P}=0.60$ ). No factors were significantly associated with $\mathrm{OS}$ in the univariate analysis: age $<60$ years $(\mathrm{HR}=1.19,95 \%$ CI $0.63-1.24, \mathrm{P}=0.59)$, primary site of disease $(\mathrm{HR}=1.17,95 \%$ CI $0.97-1.42$, $\mathrm{P}=0.11)$, MMR status $(\mathrm{HR}=0.85$, 95\% CI $0.11-6.27$, $\mathrm{P}=0.87)$; previous tumour surgery $(\mathrm{HR}=0.92,95 \% \mathrm{CI}$ $0.47-1.82, \mathrm{P}=0.81)$, RAS wild-type tumours $(\mathrm{HR}=1.47$, $95 \%$ CI $0.65-3.33, \quad \mathrm{P}=0.35)$, and lung metastasis (HR $=0.75,95 \%$ CI $0.39-1.43, \mathrm{P}=0.38)$.

Evaluation of response was performed for all patients. None of the patients showed a complete response and partial response was achieved in 3 patients. Fifty patients had stable disease. None of the patients showed a complete response. The overall response rate was $3.9 \%$, and the disease control rate was $69.7 \%$. 


\section{CEA Can Predict Fruquintinib Efficacy}

CEA was assessed after 1-2 cycles and compared with that before treatment in 34 patients. CEA increased in 18 (53\%) patients, while it decreased from baseline in $16(47 \%)$ patients. The median overall survival of the CEA-increased group and the CEA-decreased group was 10.2 months (95\% CI 7.7-12.7 months) and 17.6 months (95\% CI 12.0-23.2 months), respectively $(\mathrm{HR}=0.41,95 \% \mathrm{CI} 0.14-1.18, \mathrm{P}=0.099)$ (Figure 3$)$.

\section{Fruquintinib-Induced Hypertension}

Blood pressure was measured during each visit. A total of 37 (48.7\%) patients were diagnosed with fruquintinib-induced hypertension (hypertension group), and the remaining patients $(39,51.3 \%)$ were in the non-hypertension group. Overall, fruquintinib-induced hypertension was associated with longer OS. The median OS was 13.7 months in the hypertension group (95\% CI 9.7-17.7 months) and 7.2 months in the non-hypertension group (95\% CI $0-15.1$ months) $(\mathrm{HR}=0.59,95 \%$ CI $0.31-1.12, \mathrm{P}=0.10)$ (Figure 4).

\section{Fruquintinib-Induced Hypothyroidism}

Among the 76 patients, hypothyroidism was observed in 17 patients $(22.4 \%)$. Four patients $(5.3 \%)$ had grade 1 hypothyroidism and subclinical hypothyroidism without symptoms, while $13(17.1 \%)$ had symptomatic grade 2 hypothyroidism that thyroid hormone replacement was required.

Grade 2 hypothyroidism was observed in two out of three patients who had a PR. Patients with hypothyroidism showed a longer median OS than those with euthyroid status, and the median OS was 17.6 months (95\% CI 11.2-24.0 months) versus 10.2 months (95\% CI 5.6-14.8 months $)(\mathrm{HR}=0.62,95 \%$ CI $0.28-1.42, \mathrm{P}=0.25)$ (Figure 5).

\section{Discussion}

We conducted a retrospective review of mCRC patients who were treated with fruquintinib for later-line therapy at our institution. The reported mPFS of 5.1 months and mOS of 12.0 months in our study were slightly better

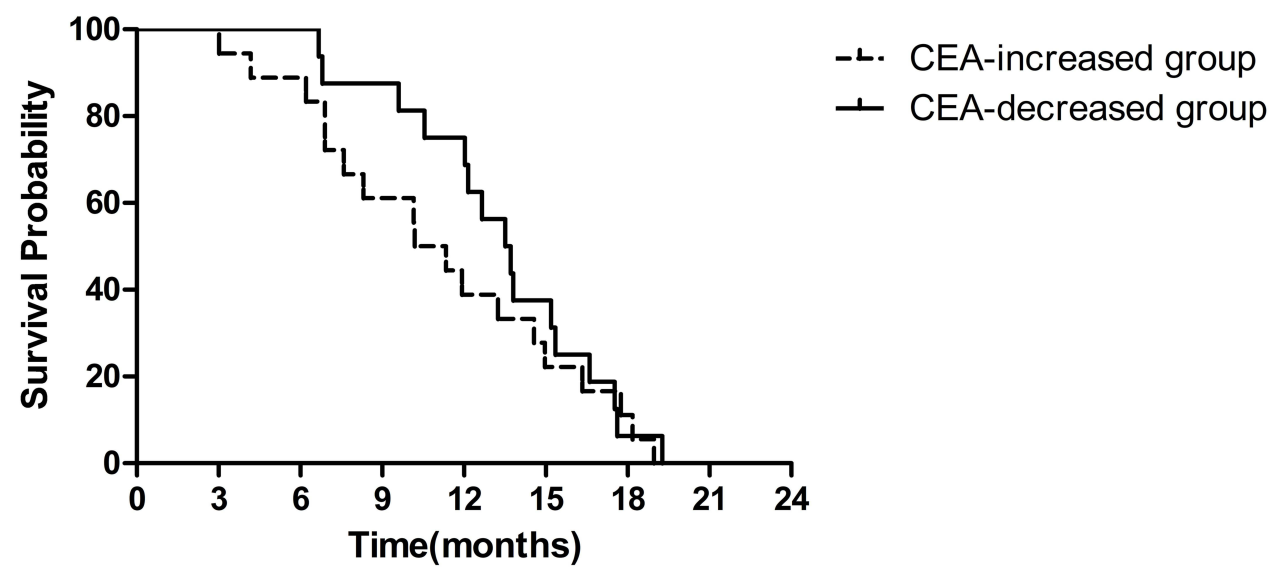

Figure 3 Kaplan-Meier survival curves of OS by CEA.

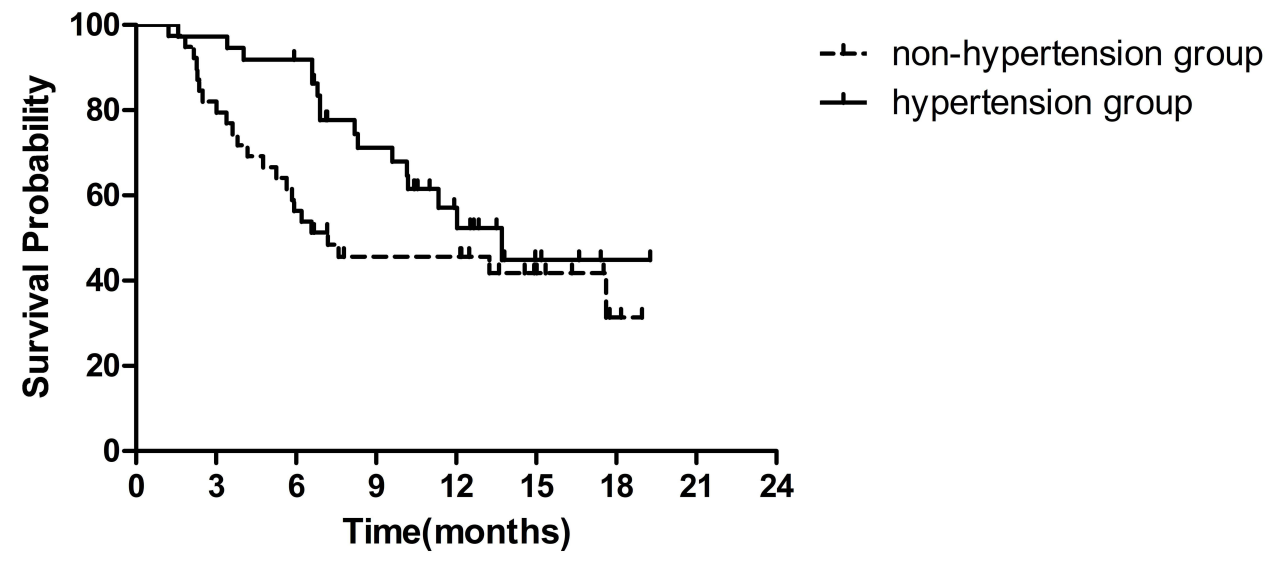

Figure 4 Kaplan-Meier survival curves of OS by fruquintinib-induced hypertension. 


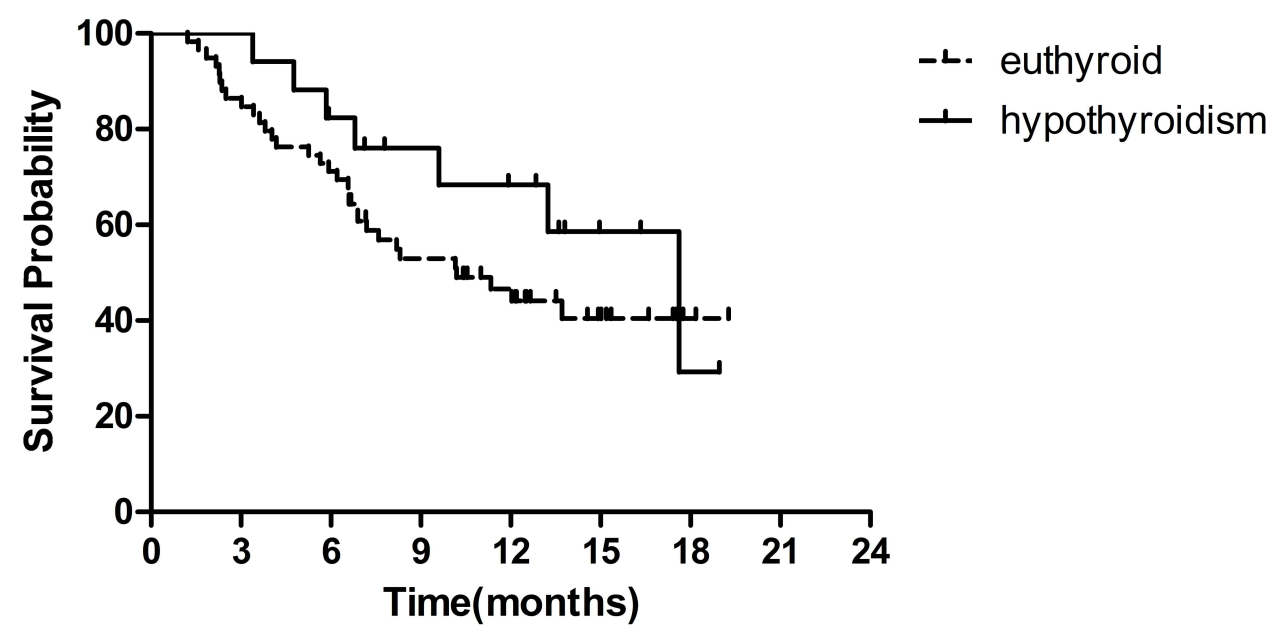

Figure 5 Kaplan-Meier survival curves of OS by fruquintinib-induced hypothyroidism.

than those demonstrated in the pivotal phase III study FRESCO, which reported an mPFS of 3.7 months and an mOS of 9.3 months. $^{5}$ This result might be attributable to differences in baseline demographics compared to our study. For instance, $10.5 \%(8 / 76)$ of the patients in our trial were took fruquintinib as first-line or second-line therapy, while all patients were treated with third-line therapy and beyond in the FRESCO trial; moreover, only $13.1 \%$ of patients in our study, compared with $27.7 \%$ in the FRESCO trial, had an ECOG PS greater than 1.

In this study, the low-dose group, which received doses starting from 3-4 mg/day for those with ECOG PS 2 or reduced from $5 \mathrm{mg} /$ day for any AE during treatment, had similar OS as the group with standard dose. Dose reduction of fruquintinib and subsequent adjustments did not affect the efficacy. In a study on regorafenib, another tyrosine kinase inhibitor similar to fruquintinib, for metastatic colorectal cancer, dose-escalation strategy was indicated as an effective approach to balance activity and tolerance. Compared to the standard-dose group, initiating a lower dose of regorafenib with weekly planned dose increases to the standard dose based on tolerability did not negatively influence efficacy and was well tolerated. ${ }^{6}$ The same treatment strategy is also applicable to fruquintinib, especially for elderly or poor patients.

Fruquintinib-induced AEs occurred in $92.1 \%$ of patients. The most frequently occurred AEs were hypertension, HFSR, proteinuria and hypothyroidism and the rates were consistent with those in the FRESCO trial $(98.6 \%) .{ }^{5}$ In this study, the most common grade 3 or 4 toxicities were HFSR, hypertension, thrombocytopenia and anaemia. This profile was generally similar to the grade 3 or 4 toxicity profile found in the FRESCO trial. ${ }^{5}$

Regardless of the hypothyroidism grade, patients with hypothyroidism showed a greater survival benefit than euthyroid patients. The therapeutic effect of regorafenib is commonly associated with thyroid dysfunction. A similar trend toward improved OS was also observed in mCRC patient treated with regorafenib who experienced the drug-related hypothyroidism. In a prospective single-centre study, the median OS was longer in patients with symptomatic hypothyroidism than in those with subclinical hypothyroidism (19.2 vs 9.4 months, $\mathrm{P}=$ 0.012 ) or with euthyroid status (19.2 vs 4.7 months, $\mathrm{P}=$ $0.001) .^{7}$ In our study, patients with fruquintinib-induced hypothyroidism showed a greater median OS than euthyroid patients, and the median OS was 17.6 months vs 10.2 months $(\mathrm{P}=0.25)$. The mechanism of TKI-induced hypothyroidism is yet to be addressed in the clinic. Studies have displayed that VEGF inhibition might take a crucial part in the development of hypothyroidism. Inhibition of VEGF with increasing TSH levels might result in capillary regression in the thyroid. ${ }^{7,8}$ Another possible mechanism for thyroid hormone synthesis interruption was associated to the presence of thyroid peroxidase antibody. ${ }^{9}$ Similarly, hypertension has been indicated as a predictive factor for longer survival. According to earlier findings, ${ }^{10,11}$ the development or worsening of hypertension suggested a response to sunitinib, another tyrosine kinase inhibitor targeting VEGFR, and favourable outcome in metastatic renal cell cancer and gastrointestinal stromal tumours. In this study, fruquintinib-induced hypertension was associated with improved clinical outcome in metastatic colorectal cancer. Patients with fruquintinib-induced hypertension had an OS of 
13.7 months, while those without fruquintinib-induced hypertension had an OS of 7.2 months $(\mathrm{P}=0.10)$. In patients with hypertension or hypothyroidism, a survival extension of more than 6 months (6.5 7.4 months) was observed, but there was no significant difference due to the small number of cases.

CEA is a common biomarker for colorectal cancer. In post hoc analyses of FRESCO patient data reported in the 2020 ASCO, the early changes in CEA during treatment as well as potential relationships with efficacy were described. In the fruquintinib group, patients with an early CEA response ( $\geq 50 \%$ decrease from baseline) had a longer median OS $(12.8$ vs 7.8 months, $\mathrm{HR}=0.45, \mathrm{p}<0.001)$ than patients without this response. ${ }^{12}$ In this study, the median overall survival of the CEA-decreased group was 7.4 months longer than that of the CEA-increased group $(\mathrm{P}=0.099)$. CEA decrease after fruquintinib treatment could be considered a potential predictor for better OS.

Although drawbacks intrinsic lies in our study as it is a retrospective analysis, and the small sample size may put a limitation on the significance of our statistical analysis, the results of this study still shed a light on the treatment of patients with fruquintinib. A real world data is a useful supplement to randomized controlled trials, which can more truly reflect the actual clinical treatment status, safety and efficacy and it has considerable clinical value.

\section{Conclusion}

In conclusion, the efficacy and safety profile of fruquintinib in the real-world practice were comparable to those found in FRESCO trial. Future studies of fruquintinib should be conducted to identify patients who tend to obtain more benefits from fruquintinib alone or in combination with other agents.

\section{Disclosure}

The authors report no conflicts of interest in this work.

\section{References}

1. Bray F, Ferlay J, Soerjomataram I, et al. Global cancer statistics 2018: GLOBOCAN estimates of incidence and mortality worldwide for 36 cancers in 185 countries. CA Cancer J Clin. 2018;68 (6):394-424. PMID: 30207593. doi:10.3322/caac.21492

2. Zheng RS, Sun KX, Zhang SW, et al. Report of cancer epidemiology in China, 2015. Zhonghua Zhong Liu Za Zhi. 2019;41(1):19-28. PMID: 30678413. doi:10.3760/cma.j.issn.0253-3766.2019.01.005

3. National Comprehensive Cancer Network. NCCN clinical practice guidelines in oncology: colon Cancer. Version 2; 2020. Available from: https://www.nccn.org/. Accessed July 31, 2021.

4. Sun Q, Zhou J, Zhang Z, et al. Discovery of fruquintinib, a potent and highly selective small molecule inhibitor of VEGFR 1, 2, 3 tyrosine kinases for cancer therapy. Cancer Biol Ther. 2014;15(12):1635-1645. PMID: 25482937. doi:10.4161/15384047.2014.964087

5. Li J, Qin S, Xu RH, et al. Effect of fruquintinib vs. placebo on overall survival in patients with previously treated metastatic colorectal cancer: the FRESCO Randomized Clinical Trial. JAMA. 2018;319 (24):2486-2496. PMID: 29946728. doi:10.1001/jama.2018.7855

6. Bekaii-Saab TS, Ou FS, Ahn DH, et al. Regorafenib dose-optimisation in patients with refractory metastatic colorectal cancer (ReDOS): a randomised, multicentre, open-label, Phase 2 study. Lancet Oncol. 2019;20(8):1070-1082. PMID: 31262657. doi:10.1016/S1470-2045(19)30272-4

7. Kim JH, Kim SY, Kim KP, et al. Regorafenib-induced hypothyroidism as a predictive marker for improved survival in metastatic or unresectable colorectal cancer refractory to standard therapies: a Prospective Single-Center Study. Target Oncol. 2019;14 (6):689-697. PMID: 31555963. doi:10.1007/s11523-019-00672-2

8. Makita N, Iiri T. Tyrosine kinase inhibitor-induced thyroid disorders: a review and hypothesis. Thyroid. 2013;23(2):151-159. PMID: 23398161. doi:10.1089/thy.2012.0456

9. Wong E, Rosen LS, Mulay M, et al. Sunitinib induces hypothyroidism in advanced cancer patients and may inhibit thyroid peroxidase activity. Thyroid. 2007;17(4):351-355. PMID: 17465866. doi:10.108 9/thy.2006.0308

10. Scartozzi M, Galizia E, Chiorrini S, et al. Arterial hypertension correlates with clinical outcome in colorectal cancer patients treated with first-line bevacizumab. Ann Oncol. 2009;20(2):227-230. PMID: 18842611. doi:10.1093/annonc/mdn637

11. Bono P, Rautiola J, Utriainen T, et al. Hypertension as predictor of sunitinib treatment outcome in metastatic renal cell carcinoma. Acta Oncol. 2011;50(4):569-573. PMID: 21208033. doi:10.3109/ 0284186X.2010.543696

12. Bai YX, Qin SK, Li J, et al. Early carcinoembryonic antigen (CEA) dynamics to predict fruquintinib efficacy in FRESCO, a $3+$ line metastatic colorectal carcinoma (mCRC) phase III trial. J Clin Oncol. 2020;38(15_suppl):abstr e16001. doi:10.1200/JCO.2020. 38.15_suppl.e16001
Cancer Management and Research is an international, peer-reviewed open access journal focusing on cancer research and the optimal use of preventative and integrated treatment interventions to achieve improved outcomes, enhanced survival and quality of life for the cancer patient.
The manuscript management system is completely online and includes a very quick and fair peer-review system, which is all easy to use. Visit http://www.dovepress.com/testimonials.php to read real quotes from published authors. 